\title{
Correlation between coronary stenosis and Toll-like receptors 2 and 4 levels in Chinese Zhuang patients with coronary heart disease
}

\author{
ZHAOHE HUANG ${ }^{1,2^{*}}$, YAN LIU ${ }^{1,2^{*}}$, LIMEI LIANG ${ }^{2}$, WENJING LIU ${ }^{2}$, SUREN R. SOORANNA ${ }^{3}$, \\ JIANJIAO MO ${ }^{2}$, LI LIU ${ }^{2}$, ZHILE LI $^{2}, \mathrm{KELA} \mathrm{LI}^{2}$ and JUN GUO ${ }^{1}$ \\ ${ }^{1}$ Department of Cardiology, The First Affiliated Hospital of Jinan University, Guangzhou, Guangdong 510630; \\ ${ }^{2}$ Department of Cardiology, Affiliated Hospital of Youjiang Medical University for Nationalities, \\ Baise, Guangxi 533000, P.R. China; ${ }^{3}$ Department of Surgery and Cancer, Imperial College London, \\ Chelsea and Westminster Hospital, London SW10 9NH, UK
}

Received February 12, 2019; Accepted June 27, 2019

DOI: $10.3892 /$ etm.2019.7805

\begin{abstract}
The present study attempted to determine the correlation of the degree of coronary artery stenosis and Tolllike receptor 2/4 (TLR2/4) levels in Chinese Zhuang patients with coronary heart disease (CHD). A total of 466 Chinese patients from the Zhuang Ethnic population diagnosed with CHD at the Department of Cardiology the Affiliated Hospital of Youjiang Medical University between January 2016 and August 2017, together with 102 control patients, were recruited for the present study. The patients with CHD were divided into three groups depending on the number of diseased arteries. The patients with CHD were also classified according to their Gensini scores. Blood liver and renal function parameters, as well as blood sugar and lipid levels were measured. ELISA was used for TLR2/4 measurements. There were no significant differences with gender, age and body mass index between the CHD and control groups. The levels of TLR2/4 in the peripheral blood of the control and CHD groups were $2.34 \pm 0.85 / 5.08 \pm 2.41$ and $5.22 \pm 3.16 / 9.33 \pm 4.92 \mathrm{ng} / \mathrm{ml}$, respectively, and the differences were significant $(\mathrm{P}<0.001)$. Analysis of the three subgroups of vessel disease indicated that the expression of TLR2/4 was progressively higher with the increase in the number of affected vessels $(\mathrm{P}<0.01)$. There were also significant differences between the mild, moderate and severe stenosis groups $(\mathrm{P}<0.01)$. A positive linear correlation between TLR2/4 and the Gensini coronary artery score
\end{abstract}

Correspondence to: Dr Suren R. Sooranna, Department of Surgery and Cancer, Imperial College London, Chelsea and Westminster Hospital, 369 Fulham Road, London SW10 9NH, UK

E-mail: s.sooranna@imperial.ac.uk

${ }^{*}$ Contributed equally

Key words: coronary heart disease, coronary artery stenosis, Gensini score, Toll-like receptor 2, Toll-like receptor 4 was identified ( $\mathrm{r}=0.508$ and 0.346 , respectively; $\mathrm{P}<0.0001)$. In conclusion, the present study determined a positive correlation between the degree of coronary artery stenosis and the expression level of TLR2/4 in the serum of Chinese Zhuang patients with CHD. Serum TLR2/4 may be used to predict the severity of CHD.

\section{Introduction}

The number of cases of coronary heart disease (CHD) is increasing worldwide. The Chinese cardiovascular disease report of 2016 indicated that the current number of cases of CHD was $\sim 11$ million (1). In 2015, the mortality rate from CHD in Chinese urban and rural residents was significantly higher than in 2014.

In recent years, several biological factors have been studied in association with CHD. Certain inflammatory factors, including tumor necrosis factor (TNF), soluble intercellular adhesion molecules (e.g. vascular cellular adhesion molecule-1 and intercellular adhesion molecule-1), matrix metalloproteinases, hypersensitive C-reactive protein (hs-CRP) and interleukin-6 (IL-6) have all been extensively studied in different groups of patients with CHD (2). It has been indicated that Toll-like receptors (TLRs) are involved in the occurrence and development of coronary atherosclerosis (3), implying that TLRs may be potential novel targets for intervention of atherosclerosis. A previous study suggested that the protein expression of TLR 2 and TLR 4 was involved in the regulation of cardiovascular function in mice (4).

The Han and Zhuang ethnic groups make up the largest proportion of the population of the Guangxi Zhuang Autonomous Region of China (62 and 32\%, respectively). Previously, a large number of studies have focused on diseases in these two groups within the population with several genetic differences, including the prevalence of genes linked to hyperlipidemia and 3'APOB-VNTR polymorphisms, and other differences, including the presence of many pharmacogenetic variants and drug detoxification mechanisms, having been identified (5-7). In this light, the present study was performed 
to explore diagnostic biomarkers of coronary artery stenosis in patients of the ethnic Zhuang group with CHD and to validate potential correlations between the expression levels of TLR2/4 in the circulatory system. The results indicated that the measurement of TLR2/4 provides a tool for early clinical diagnosis and a simple way of assessing the severity of CHD in such patients.

\section{Patients and methods}

Patients. The present study complied with the Declaration of Helsinki and was approved by the Ethics Committee Review Board of the Affiliated Hospital of Youjiang Medical University for Nationalities (Baise, China). Written informed consent was obtained from each of the patients enrolled in the study. The present study is a prospectively planned observational study on 568 subjects admitted to The Affiliated Hospital of Youjiang Medical University (Baise, China) between January 2016 and August 2016 who underwent coronary angiography. They were divided into two groups according to the results of coronary angiography, namely the CHD group (with $\geq 50 \%$ stenosis) and a control group (with no stenosis). They were all from the Zhuang ethnic group. Patients with CHD (males, $n=297$; females, $n=169$; age, $38-81$ years; average age, $59.76 \pm 10.40$ years). In addition, 102 healthy individuals were recruited during the same time period (males, $n=57$; females, $n=45$; age, $41-72$ years; average age, $62.15 \pm 10.32$ years).

The exclusion criteria for the CHD group of patients were as follows: Participants with rheumatic heart disease, dilated cardiomyopathy, congenital heart disease, patients undergoing intravenous thrombolysis, coronary stenting and coronary artery bypass grafting, systemic or local severe infection, auto-immunologic and blood system disease, severe kidney or liver disease or malignant disease. The healthy control group of patients were recruited from the Out-patients area within the hospital including the renal and respiratory departments. None of the patients included in the study had severe symptoms and they were discharged within $12 \mathrm{~h}$ of admission. All patients who had severe illnesses and were kept $>12 \mathrm{~h}$ and those who had a history of heart disease were also excluded from the study.

The CHD cases were further divided into the following subgroups according to the number of diseased vessels: i) Cases with single-vessel disease $(n=127 ; 81$ males and 46 females; average age, $58.56 \pm 10.41$ years), ii) cases with double-vessel disease $(n=243 ; 156$ males and 87 females; average age, $60.97 \pm 10.26$ years) and iii) cases with multi-vessel disease $(n=96 ; 55$ males and 41 females; average age, $58.25 \pm 10.46$ years). The quantitative analysis of the severity of coronary artery disease was performed according to the Gensini integral (8), based on which the patients were stratified into three groups: i) Mild stenosis group $(\mathrm{n}=126$; 55 males and 71 females; average age, 58.88 \pm 10.84 years), ii) moderate stenosis group $(n=261 ; 175$ males and 86 females; average age, $60.21 \pm 10.26$ years) and iii) severe stenosis group ( $\mathrm{n}=79 ; 49$ males and 30 females; average age, $59.65 \pm 10.70$ years).

There were no significant differences in gender, age, smoking habits, alcohol consumption, body mass index (BMI), blood pressure and incidence of diabetes between the CHD and control groups. None of the cases included in the present study had any severe infections, immune system diseases, connective tissue disease, blood system diseases, malignant tumors, liver and kidney malfunctions, recent major surgeries or severe trauma.

Methods. All subjects were routinely fasted for at least $8 \mathrm{~h}$ prior to obtainment of blood samples for analysis. These included tests for glucose, lipid proteins, liver and renal function parameters. The subjects were also subjected to the oral glucose tolerance test. The protein concentrations of TLR2 and TLR4 in the serum were detected using RT-6000 ELISA kits (Rayto Life and Analytical Sciences Co., Ltd.). TLR2 and TLR4 kits were obtained from Wuhan Huamei Biological Engineering Co., Ltd. The intra- and inter-assay coefficient of variation for the ELISA kits were determined to be $<5 \%$ and $<15 \%$, respectively. The detection limits of the ELISAs were 0.54 and $1.38 \mathrm{ng} / \mathrm{ml}$ for TLR2 and TLR4, respectively.

Blood was collected aseptically from the caudal vein by venipuncture into one of three vacutainer tubes containing either sodium heparin, sodium citrate or EDTA. These tubes were immediately centrifuged at $375 \mathrm{x}$ g for $15 \mathrm{~min}$ in bench centrifuge at $4^{\circ} \mathrm{C}$ and samples were prepared or analyzed within $45 \mathrm{~min}$ of collection. Aliquots were frozen at $-80^{\circ} \mathrm{C}$ for ELISA.

All patients underwent a routine coronary angiography in strict accordance with the operating procedures. The coronary intervention was an adaptation of the Judkins technique (8). The right radial artery or femoral artery paths were followed during the operation, which was performed by at least two interventional cardiovascular physicians. The coronary angiography results were recorded in detail together with the degree of stenosis and blood flow of the coronary lesions. When the degree of coronary artery stenosis of at least one vessel in the left main stem, the left anterior descending branch, the left circumflex branch, the right coronary artery or its major branches was $>50 \%$, this was diagnosed as CHD. According to the number of vascular lesions involved in the disease, the patients were classified into i) the single-vessel disease group, ii) the double-vessel disease group or iii) the multi-vessel disease group. The Gensini score was determined according to the degree of each lesion of vessel stenosis (9).

Statistical analysis. SPSS 17.0 software (SPSS, Inc.) was used for statistical analysis. Measurement data are expressed as the mean \pm standard deviation. Comparisons between multiple groups were performed using single-factor analysis of variance followed by Tukey's post hoc test. Comparisons between two groups were performed using the unpaired t-test, while the Shapiro-Wilk test was used to check whether the data were normally distributed. $\mathrm{P}<0.05$ was considered to indicate statistical significance. The correlation between the severity of coronary artery stenosis and TLR 2 or TLR4 was determined using Pearson's correlation analysis. Comparison between the clinicopathological parameters of the patients in the CHD and control groups were compared using the paired Chi-square test. 
Table I. Characteristics of the control subjects and CHD patients in the present study.

\begin{tabular}{|c|c|c|c|c|}
\hline Parameter & Control subjects $(n=102)$ & CHD patients $(n=466)$ & $\chi^{2} /$ t-value & P-value \\
\hline Age (years) & $62.15 \pm 10.32$ & $59.76 \pm 10.40$ & 1.066 & 0.286 \\
\hline BMI $\left(\mathrm{kg} / \mathrm{m}^{2}\right)$ & $21.39 \pm 2.58$ & $22.17 \pm 2.91$ & 0.844 & 0.399 \\
\hline Sex & & & 2.197 & 0.138 \\
\hline Male & $57(55.9)$ & $297(63.7)$ & & \\
\hline Female & $45(44.1)$ & $169(36.3)$ & & \\
\hline Smoking & & & 0.376 & 0.539 \\
\hline Yes & $32(31.4)$ & $161(34.5)$ & & \\
\hline No & $70(68.6)$ & $305(65.5)$ & & \\
\hline Alcohol consumption & & & 1.980 & 0.159 \\
\hline Yes & $27(26.5)$ & 94 (20.2) & & \\
\hline No & $75(73.5)$ & $372(79.8)$ & & \\
\hline Hypertension & & & 2.219 & 0.136 \\
\hline Yes & $32(31.4)$ & $183(39.3)$ & & \\
\hline No & $70(68.6)$ & $283(60.7)$ & & \\
\hline Type 2 diabetes mellitus & & & 0.122 & 0.727 \\
\hline Yes & $11(10.8)$ & $56(12.0)$ & & \\
\hline No & $91(89.2)$ & $410(88.0)$ & & \\
\hline
\end{tabular}

Values are expressed as n (\%). BMI, body mass index; CHD, coronary heart disease.

\section{Results}

Characteristics of patients. The CHD and the control groups were not significantly different in many of the general characteristics, including gender, age, smoking and drinking habits, BMI and incidence of hypertension and diabetes, indicating that the baseline characteristics of the subjects were consistent and comparable between the two groups (Table I).

$T L R$ protein levels in patient groups. Comparison of the protein expression levels of TLR 2 and TLR4 between the CHD and the control groups was performed by ELISA measurements in the sera of all 568 patients. The results indicated that the respective concentrations of TLR 2 and TLR4 in the serum were $5.22 \pm 3.16$ and $9.33 \pm 4.92 \mathrm{ng} / \mathrm{ml}$ in CHD patients, and the values obtained for control subjects were significantly lower at $2.34 \pm 0.85$ and $5.08 \pm 2.41 \mathrm{ng} / \mathrm{ml}$, respectively $(\mathrm{P}<0.001$; Table II and Fig. 1). This suggests that the serum concentrations of TLR 2 and TLR 4 are closely linked to the occurrence and development of CHD in the ethnic Zhuang population.

Comparisons of the expression levels of TLR 2 or TLR4 between different groups according to the number of coronary lesions were also performed. The concentration of TLR2 in the serum was $3.05 \pm 1.38,5.67 \pm 3.12$ and $6.94 \pm 3.41 \mathrm{ng} / \mathrm{ml}$, in the single-, double- and multi-lesion group, respectively. The corresponding values for TLR 4 in the three groups were $6.75 \pm 3.84,9.89 \pm 4.72$ and $11.34 \pm 5.3 \mathrm{ng} / \mathrm{ml}$, respectively ( $\mathrm{P}<0.01$; Table III and Fig. 2). The highest expression of TLR was in the multi-vessel lesion group, followed by the two-vessel lesion group and the lowest expression was in the single-vessel lesion group.
Table II. Concentrations of TLR2 and TLR4 in control and CHD patients.

\begin{tabular}{lcccc}
\hline & $\begin{array}{c}\text { Control } \\
\text { subjects } \\
(\mathrm{n}=102)\end{array}$ & $\begin{array}{c}\text { CHD } \\
\text { patients } \\
(\mathrm{n}=466)\end{array}$ & t-value & P-value \\
Protein & $2.34 \pm 0.85$ & $5.22 \pm 3.16$ & 17.039 & $<0.001$ \\
\hline TLR2 $(\mathrm{ng} / \mathrm{ml})$ & $2.08 \pm 2.41$ & $9.33 \pm 4.92$ & 12.892 & $<0.001$ \\
\hline TLR4 $(\mathrm{ng} / \mathrm{ml})$ & 5.08 & & & \\
\hline
\end{tabular}

CHD, coronary heart disease; TLR, Toll-like receptor.

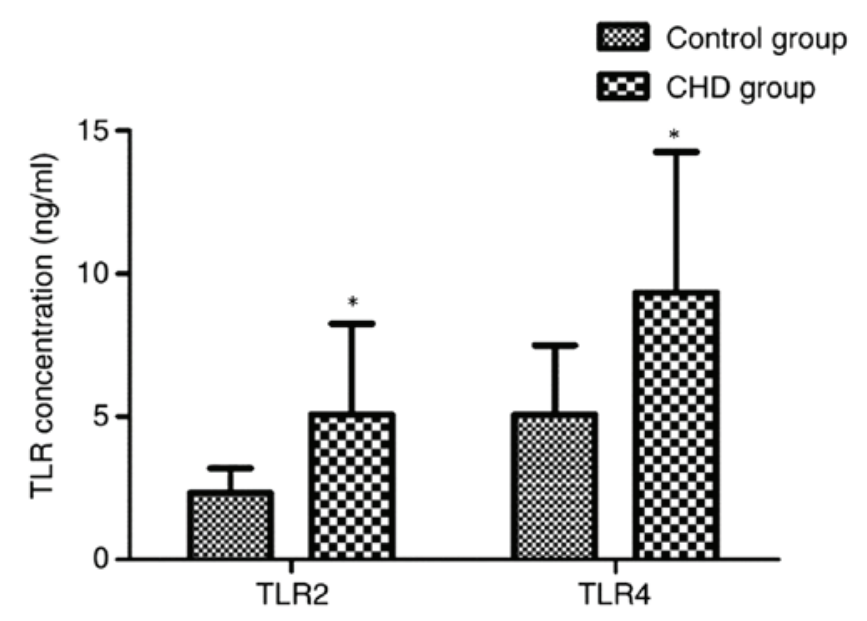

Figure 1. ELISA confirmed the significant increase in the serum levels of TLR2 and TLR4 in CHD patients compared with those of control patients. Values are expressed as the mean \pm standard error of the mean. ${ }^{*} \mathrm{P}<0.01$. TLR, Toll-like receptor; CHD, coronary heart disease. 
Table III. Association between the levels of TLR2 or TLR4 in patients with coronary heart disease and the number of coronary lesions.

\begin{tabular}{lcccc}
\hline Protein & $\begin{array}{c}\text { Single-vessel } \\
\text { lesion }(\mathrm{n}=127)\end{array}$ & $\begin{array}{c}\text { Two-vessel } \\
\text { lesions }(\mathrm{n}=243)\end{array}$ & $\begin{array}{c}\text { Multi-vessel } \\
\text { lesions }(\mathrm{n}=96)\end{array}$ & F-value \\
\hline TLR2 $(\mathrm{ng} / \mathrm{ml})$ & $3.05 \pm 1.38$ & $5.67 \pm 3.12^{\mathrm{a}}$ & $6.94 \pm 3.41^{\mathrm{a}, \mathrm{b}}$ & 108.436 \\
TLR4 $(\mathrm{ng} / \mathrm{ml})$ & $6.75 \pm 3.84$ & $9.89 \pm 4.72^{\mathrm{a}}$ & $11.34 \pm 5.30^{\mathrm{a}, \mathrm{b}}$ & 58.775 \\
\hline
\end{tabular}

${ }^{a} \mathrm{P}<0.01$ compared with the single-vessel lesion group; ${ }^{b} \mathrm{P}<0.01$ compared with the two-vessel lesions group. TLR, Toll-like receptor.

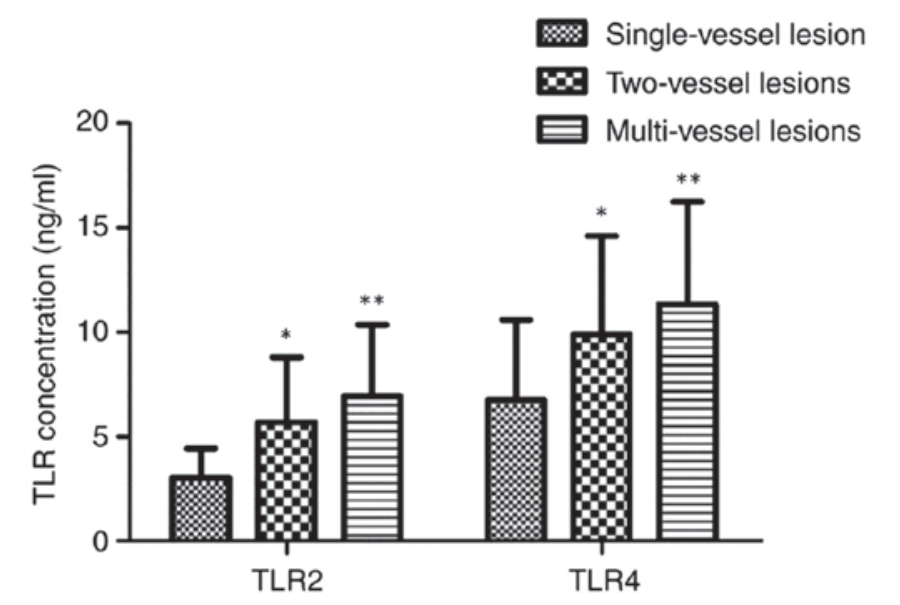

Figure 2. Protein expression of TLR2 and TLR4 in the serum of patients with single-vessel lesion, two-vessel lesions and multi-vessel lesions determined by ELISA. Values are expressed as the mean \pm standard error of the mean. ${ }^{*} \mathrm{P}<0.01$ vs. the single-vessel lesion group and ${ }^{* * *} \mathrm{P}<0.01$ vs. the two-vessel lesions group. TLR, Toll-like receptor.

The expression levels of TLR 2 and TLR4 protein between the subgroups by severity of coronary artery stenosis were $2.67 \pm 1.06,5.81 \pm 2.99$ and $7.32 \pm 3.44 \mathrm{ng} / \mathrm{ml}$, respectively, for the mild, moderate and severe stenosis groups. The corresponding values for TLR4 in the three groups were $6.38 \pm 3.17,9.80 \pm 4.74$ and $12.48 \pm 5.32 \mathrm{ng} / \mathrm{ml}$, respectively. The levels of TLR2 and TLR4 in the severe stenosis group were markedly higher than those in the mild and moderate stenosis groups, and the TLR2 and TLR4 levels in the moderate stenosis group were higher than those in the mild stenosis group $(\mathrm{P}<0.01$; Table IV and Fig. 3). These results imply that in the Zhuang ethnic group of patients, the degree of coronary artery stenosis is associated with the circulatory levels of TLR2 and TLR4, with higher levels observed in the more severe cases of CHD.

Correlation between TLR expression and vessel stenosis. Positive correlations between the expression levels of TLR2 or TLR4 and the coronary Gensini score were determined using Pearson's correlation analysis. From this, the R-values were calculated to be 0.508 and 0.346 for TLR2 and TLR4, respectively $(\mathrm{P}<0.0001$; Fig. $4 \mathrm{~A}$ and $\mathrm{B})$. It appeared that in Zhuang patients with CHD, the severity of coronary artery lesions is correlated with the plasma levels of TLR2 or TLR4 and that the patients with higher plasma concentrations of TLR2 and TLR4 have a higher degree of severity of coronary artery lesions.

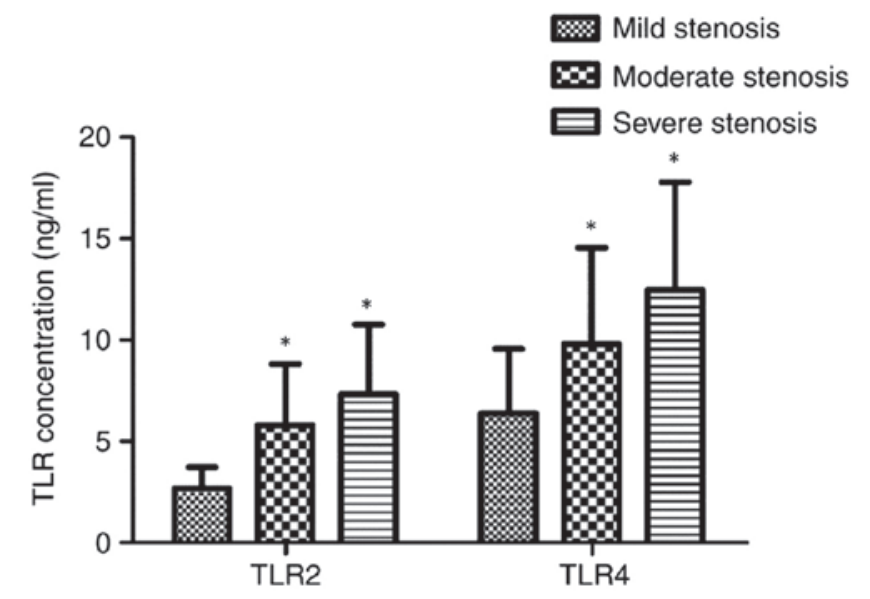

Figure 3. Protein expression levels of TLR 2 and TLR4 in the serum of patients with mild stenosis, moderate stenosis and severe stenosis determined by ELISA. Values are expressed as the mean \pm standard error of the mean. ${ }^{*} \mathrm{P}<0.01$ vs. the mild stenosis group. TLR, Toll-like receptor.

\section{Discussion}

CHD that occurs due to coronary vascular atherosclerosis proceeds to luminal stenosis and/or obstruction, or coronary artery blood vessel function changes (spasms) and myocardial tissue ischemia, hypoxia or necrosis. The associated pathological changes result in atherosclerotic coronary blood vessels, which in turn form stable or unstable plaques. These events are the preclinical phase of coronary disease, frequently leading to the rupture of plaques or lumen invasion, which in turn leads to incomplete or complete vascular occlusion. During the process of development and occurrence of CHD, a large number of inflammatory factors are involved, including changes in the levels of TLR2, TLR4, hs-CRP, IL-6, nitric oxide, myeloperoxidase, TNF, CD40 and complement factors. These may be considered as important inflammatory markers, which trigger and aggravate the inflammatory response and participate in the occurrence and development of CHD $(10,11)$.

TLRs are members of the natural immune response and are widely distributed in the total genome (12) TLR2 and TLR4 are also involved in the occurrence and development of coronary atherosclerosis. TLR2 and TLR4 are mainly distributed on the cell surface and are able to recognize lipids. After its isomerization, TLR2 it is able to recognize the peptides, lipoprotein and mycoplasma of gram-positive bacteria. During atherosclerosis and subsequent hardening of the arteries, TLR4 leads to the activation of inflammatory signaling pathways, which in turn initiate 
Table IV. Association between the levels of TLR 2 or TLR4 in patients with coronary heart disease and the severity of coronary artery stenosis.

\begin{tabular}{lccccr}
\hline Protein & $\begin{array}{c}\text { Mild stenosis } \\
(\mathrm{n}=126)\end{array}$ & $\begin{array}{c}\text { Moderate stenosis } \\
(\mathrm{n}=261)\end{array}$ & $\begin{array}{c}\text { Severe stenosis } \\
(\mathrm{n}=79)\end{array}$ & F-value & P-value \\
\hline TLR2 $(\mathrm{ng} / \mathrm{ml})$ & $2.67 \pm 1.06$ & $5.81 \pm 2.99^{\mathrm{a}}$ & $7.32 \pm 3.44^{\mathrm{a}, \mathrm{b}}$ & 162.697 & $<0.01$ \\
TLR4 $(\mathrm{ng} / \mathrm{ml})$ & $6.38 \pm 3.17$ & $9.80 \pm 4.74^{\mathrm{a}}$ & $12.48 \pm 5.32^{\mathrm{a}, \mathrm{bb}}$ & 81.155 & $<0.01$ \\
\hline
\end{tabular}

${ }^{a} \mathrm{P}<0.01$ compared with the mild stenosis group; ${ }^{\mathrm{b}} \mathrm{P}<0.01$ compared with the moderate stenosis group. TLR, Toll-like receptor.

A $y=30.93+5.72 * x$

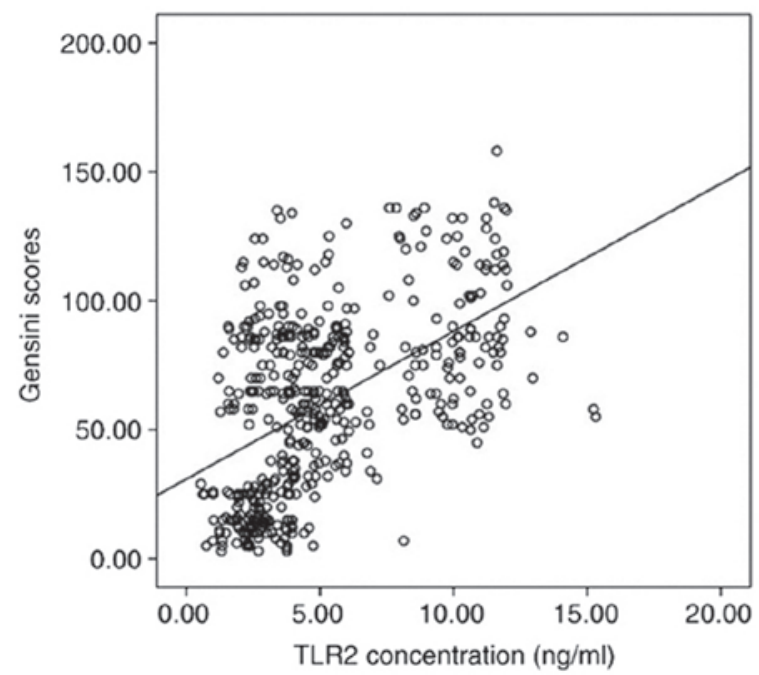

B $y=37.42+2.5 * x$

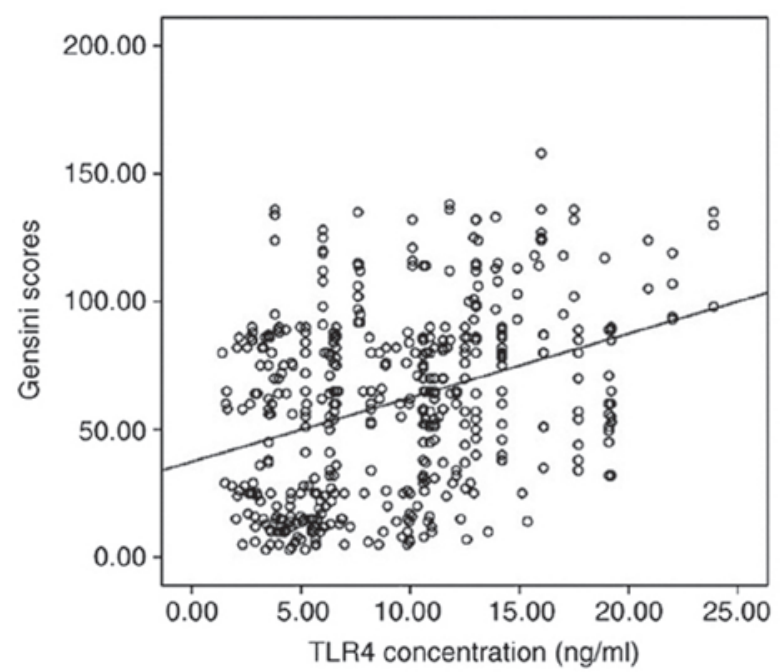

Figure 4. Analysis of the correlation between coronary Gensini scores and the expression levels of (A) TLR2 (slope, y=30.93+5.72x) and (B) TLR4 (slope, $y=37.42+2.5 x)$. TLR, Toll-like receptor.

the start induction of intracellular signal transduction. This causes the activation of $\mathrm{NF}-\mathrm{\kappa B}(13)$, which translocates into the nucleus, promoting activation of the mitogen-activated protein kinase pathway. Upregulation of hs-CRP, TNF- $\alpha$ and IL-6 follows (14), leading to the induction of macrophage infiltration and activation. This aggravates the inflammatory response, promoting inflammatory activation of atherosclerotic plaque, which may result in plaque rupture.

TLR4 may also identify lipopolysaccharides (LPS), which occur in the outer membrane of Gram-negative bacteria, and this promotes the development of atherosclerosis (15). Studies on TLR2 and TLR4 suggested that these receptors are involved in signaling pathways mediating inflammation that may lead to the occurrence and development of coronary artery disease (16). It was also indicated that in male patients with CHD, TLR4 mRNA levels were positively correlated with the severity of coronary stenosis (17). In addition, a study on CHD patients of the Han ethnic group suggested differences in TLR 2 and TLR4 expression between genders and these differences were linked to the levels of sex hormone (18).

A previous study indicated that the urinary albumin creatinine ratio and cystatin $\mathrm{C}$ levels have a certain predictive value for the degree of coronary artery stenosis (19). The aim of the present study was mainly to investigate the correlation between the severity of coronary artery stenosis and the expression levels of TLR2 and TLR4 in circulating blood in patients of the ethnic Zhuang group with CHD. The results suggested that, compared with those in the healthy control group, the levels of TLR2 and TLR4 were significantly increased in patients with CHD, indicating that TLR2 and TLR4 may be involved in the occurrence and development of coronary atherosclerosis. Whether the patients were classified according to the number of diseased vessels or to the coronary Gensini scores, the severity of CHD was observed to correlate with these parameters. The concentrations of TLR2 and TLR4 in circulating blood appeared to have a certain predictive value for the degree of coronary artery stenosis in patients with CHD. In patients with $>90 \%$ stenosis, an association between a high degree of stenosis and reduced TLR4 responsiveness has been reported (20).

In addition, the present results indicated that the concentrations of TLR2 and TLR4 were higher in patients with two-vessel lesions, multi-vessel lesions, moderate stenosis and severe stenosis, particularly in multi-vessel lesions and severe stenosis. This means that higher concentrations of TLR2 and TLR4 in circulating blood are associated with more severe coronary artery stenosis, resulting in a larger number of diseased vessels. Previous studies suggested that TLR4 mediated the release of TNF- $\alpha$ and IL- 6 and that the release of pro-inflammatory cytokines was higher in patients with unstable angina or acute myocardial infarction than in those 
with stable angina or healthy controls (21-23). The expression of TLR4 on monocytes is associated with TNF- $\alpha$ and IL-6 release after LPS stimulation, with TLR4 participating in the occurrence and development of CHD via the inflammatory response (22). In addition, it was reported that the surface expression of TLR2 and TLR4 on circulating monocytes increased markedly in acute myocardial infarction and unstable patients with angina compared with that in healthy control subjects $(21,24)$. However, these findings have yet to be fully explained and may be linked to genetic factors.

In the present study, the expression levels of TLR2 and TLR4 in the circulating blood of CHD patients were significantly higher than those in subjects without CHD. Higher expression of TLR2 and TLR4 in the serum was associated with larger and more numerous coronary artery lesions, resulting in a higher severity of coronary artery stenosis. Thus, serum TLR2 and TLR4 levels in CHD patients may have predictive value regarding the severity of coronary artery disease stenosis. In future studies, it may be possible to measure the concentrations of TLR 2 and TLR4 more accurately and rapidly in order to increase their usefulness as predictive markers.

Several subtypes of CHD (including myocardial infarction, stable angina pectoris and unstable angina pectoris) were included in the present study, but the numbers of patients in these groups were relatively small and analysis of their serum TLR2 and TLR4 levels would not have been meaningful. In addition, the size of the control group in the present study was relatively small. In future studies, patients from several hospitals may be recruited in order to overcome the limitations of the present study.

\section{Acknowledgements}

Not applicable.

\section{Funding}

This study was supported by a grant from the Baise Science and Technology Cooperation Project Foundation of Guangxi Province, China (grant no. 20150819).

\section{Availability of data and materials}

The datasets used and/or analyzed during the current study are available from the corresponding author on reasonable request.

\section{Authors' contributions}

$\mathrm{ZH}$, WL, LLiang, YL and JM were involved in acquisition, analysis or interpretation of the data. ZH, YL, JM, KL, SRS, LLiu and ZL contributed to the design and the conception of the study and interpretation of the data. $\mathrm{ZH}, \mathrm{YL}, \mathrm{WL}$ and JG conceived of the study and participated in its design and coordination. ZH, YL and JG drafted the manuscript. All authors were involved in drafting/revising and approving the manuscript.

\section{Ethics approval and consent to participate}

This study was approved by the Ethics Committee of the Affiliated Hospital of Youjiang Medical University for Nationalities (Baise, China), in accordance with the Declaration of Helsinki. All participants provided written informed consent to participate in this study.

\section{Patient consent for publication}

Not applicable.

\section{Competing interests}

The authors declare that they have no competing interests.

\section{References}

1. Wang Z, Wang X, Chen Z, Zhang L and Zhu M: Distribution of high-sensitivity $\mathrm{C}$-reactive protein and its relationship with other cardiovascular risk factors in the middle-aged chinese population. Int J Environ Res Public Health 13: pii: E872, 2016.

2. Sepehri ZS, Masoomi M, Ruzbehi F, Kiani Z, Nasiri AA, Kohan F, Sheikh Fathollahi M, Kazemi Arababadi M, Kennedy D and Asadikaram GA: Comparison of serum levels of IL-6, IL-8, TGF- $\beta$ and TNF- $\alpha$ in coronary artery diseases, stable angina and participants with normal coronary artery. Cell Mol Biol (Noisy-le-grand) 64: 1-6, 2018

3. Kuwahata S, Fujita S, Orihara K, Hamasaki S, Oba R, Hirai H, Nagata K, Ishida S, Kataoka T, Oketani N, et al: High expression level of Toll-like receptor 2 on monocytes is an important risk factor for arteriosclerotic disease. Atherosclerosis 209: 248-254, 2010.

4. Okun E, Griffioen KJ, Rothman S, Wan R, Cong WN, De Cabo R, Martin-Montalvo A, Levette A, Maudsley S, Martin B, et al: Toll-like receptors 2 and 4 modulate autonomic control of heart rate and energy metabolism. Brain Behav Immun 36: 90-100, 2014.

5. Ruixing Y, Fengping H, Shangling P, Dezhai Y, Weixiong L, Tangwei L, Yuming C, Jinzhen W, Limei Y and Jiandong H: Prevalence of hyperlipidemia and its risk factors for the middle-aged and elderly in the Guangxi Hei Yi Zhuang and Han populations. J Investig Med 54: 191-200, 2006.

6. Ruixing Y, Guangqin C, Yong W, Weixiong L, Dezhai Y and Shangling P: Effect of the 3'APOB-VNTR polymorphism on the lipid profiles in the Guangxi HeiYi Zhuang and Han populations. BMC Med Genet 8: 45, 2007.

7. Li J, Guo C, Yan M, Niu F, Chen P, Li B and Jin T: Genetic polymorphisms in very important pharmacogenomic variants in the Zhuang ethnic group of Southwestern China: A cohort study in the Zhuang population. Medicine (Baltimore) 97: e0559, 2018.

8. Judkins MP: Percutaneous transfemoral selective coronary arteriography. Radiol Clin North Am 6: 467-492, 1968 (In Portuguese).

9. Gensini GG: A more meaningful scoring system for determining the severity of coronary heart disease. Am J Cardiol 51: 606, 1983.

10. Harrington RA: Targeting inflammation in coronary artery disease. N Engl J Med 377: 1197-1198, 2017.

11. Li H, Sun K, Zhao R, Hu J, Hao Z, Wang F, Lu Y, Liu F and Zhang Y: Inflammatory biomarkers of coronary heart disease. Front Biosci (Landmark Ed) 22: 504-515, 2017.

12. McCoy KL: Interaction between cannabinoid system and toll-like receptors controls inflammation. Mediators Inflamm 2016: 5831315, 2016.

13. Sugiyama K, Muroi M, Kinoshita M, Hamada O, Minai Y, Sugita-Konishi Y, Kamata Y and Tanamoto K: NF- $\kappa B$ activation via MyD88-dependent Toll-like receptor signaling is inhibited by trichothecene mycotoxin deoxynivalenol. J Toxicol Sci 41: 273-279, 2016.

14. Jia SJ, Niu PP, Cong JZ, Zhang BK and Zhao M: TLR4 signaling: A potential therapeutic target in ischemic coronary artery disease. Int Immunopharmacol 23: 54-59, 2014.

15. Zhang Y, El-Far M, Dupuy FP, Abdel-Hakeem MS, He Z, Procopio FA, Shi Y, Haddad EK, Ancuta P, Sekaly RP and Said EA: HCV RNA activates APCs via TLR7/TLR8 while virus selectively stimulates macrophages without inducing antiviral responses. Sci Rep 6: 29447, 2016.

16. Yu L and Feng Z: The role of toll-like receptor signaling in the progression of heart failure. Mediators Inflamm 2018: 9874109, 2018. 
17. Guven M, Ismailoglu Z, Batar B, Unal S, Onaran I, Karadag B and Ongen $Z$ : The effect of genetic polymorphisms of TLR2 and TLR4 in Turkish patients with coronary artery disease. Gene 558: 99-102, 2015.

18. Lashkari BS, Shahana S and Anumba DO: Toll-like receptor 2 and 4 expression in the pregnant and non-pregnant human uterine cervix. J Reprod Immunol 107: 43-51, 2015.

19. de Boer RA, Nayor M, deFilippi CR, Enserro D, Bhambhani V, Kizer JR, Blaha MJ, Brouwers FP, Cushman M, Lima JAC, et al: Association of cardiovascular biomarkers with incident heart failure with preserved and reduced ejection fraction. JAMA Cardiol 3: 215-224, 2018.

20. Versteeg D, Hoefer IE, Schoneveld AH, de Kleijn DP, Busser E, Strijder C, Emons M, Stella PR, Doevendans PA and Pasterkamp G: Monocyte toll-like receptor 2 and 4 responses and expression following percutaneous coronary intervention: Association with lesion stenosis and fractional flow reserve. Heart 94: 770-776, 2008

21. Methe H, Kim JO, Kofler S, Weis M, Nabauer M and Koglin J: Expansion of circulating Toll-like receptor 4-positive monocytes in patients with acute coronary syndrome. Circulation 111: 2654-2661, 2005.
22. Satoh M, Shimoda Y, Maesawa C, Akatsu T, Ishikawa Y, Minami Y, Hiramori K and Nakamura M: Activated toll-like receptor 4 in monocytes is associated with heart failure after acute myocardial infarction. Int J Cardiol 109: 226-234, 2006.

23. Liuzzo G, Angiolillo DJ, Buffon A, Rizzello V, Colizzi C, Ginnetti F, Biasucci LM and Maseri A: Enhanced response of blood monocytes to in vitro lipopolysaccharide-challenge in patients with recurrent unstable angina. Circulation 103: 2236-2241, 2001.

24. Ashida K, Miyazaki K, Takayama E, Tsujimoto $\mathrm{H}$, Ayaori M, Yakushiji T, Iwamoto N, Yonemura A, Isoda K, Mochizuki H, et al: Characterization of the expression of TLR2 (toll-like receptor 2) and TLR4 on circulating monocytes in coronary artery disease. J Atheroscler Thromb 12: 53-60, 2005. 